\title{
Sistemas virtuais de aprendizagem personalizados: estilos de aprendizagem, aprendizagem organizacional e usabilidade
}

\section{Virtual personalized learning systems: learning styles, organizational learning and usability}

DOI: $10.46919 / \operatorname{archv1n4-001}$

Recebimento dos originais: 10/05/2020

Aceitação para publicação: 30/06/2020

\author{
Danielli Cossul \\ Graduanda em Psicologia pela Universidade de Santa Cruz do Sul (UNISC) \\ Instituição: Universidade de Santa Cruz do Sul (UNISC) \\ Endereço: Avenida Independência, 2293 Bairro Universitário, Santa Cruz do Sul - RS, 96815-900 \\ E-mail: daniellicossul@m2.unisc.br \\ Marcia de Bastos Braatz \\ Psicóloga graduada pela Universidade de Santa Cruz do Sul (UNISC) \\ Instituição: Universidade de Santa Cruz do Sul (UNISC) \\ Endereço: Avenida Independência, 2293 Bairro Universitário, Santa Cruz do Sul - RS, \\ 96815-900 \\ E-mail: m.braatz.psi@gmail.com

\section{Marcus Vinicius Castro Witczak} \\ Doutor em Psicologia pela Pontifícia Universidade Católica do Rio Grande do Sul (PUCRS) \\ Instituição: Universidade de Santa Cruz do Sul (UNISC) \\ Endereço: Avenida Independência, 2293 Bairro Universitário, Santa Cruz do Sul - RS, 96815-900 \\ E-mail: marcus@unisc.br

\section{Rejane Frozza} \\ Doutora em Ciência da Computação pela Universidade Federal do Rio Grande do Sul (UFRGS) \\ Instituição: Universidade de Santa Cruz do Sul (UNISC) \\ Endereço: Avenida Independência, 2293 Bairro Universitário, Santa Cruz do Sul - RS, 96815-900 \\ E-mail: frozza@unisc.br

\section{Liane Mählmann Kipper} \\ Doutora em Engenharia da Produção pela Universidade Federal de Santa Catarina (UFSC) \\ Instituição: Universidade de Santa Cruz do Sul (UNISC) \\ Endereço: Avenida Independência, 2293 Bairro Universitário, Santa Cruz do Sul - RS, 96815-900 \\ E-mail: liane@unisc.br
}

\section{RESUMO}

A busca pela inovação em espaços organizacionais, com o uso de Ambientes Virtuais de Aprendizagem personalizados, implica explicitar os processos responsáveis pela modelização comportamental que repercute nas experiências de colaboradores(as). Nesse sentido, o objetivo é compreender as influências das teorias clássicas de aprendizagem do século XX sobre as teorias da administração. Objetivo este complementado por explorar como auxiliar o processo de aprendizagem organizacional com o uso de sistemas virtuais de aprendizagem que se adaptam aos estilos de aprendizagem dos usuários e com uma 
interface amigável. O método adotado foi a revisão narrativa da literatura coletada a partir do levantamento bibliográfico sobre os campos teorias da aprendizagem e escolas de administração. Apresenta-se como principal resultado, uma análise dialógica acerca dos discursos epistemológicos que fundem os pressupostas das escolas de administração de recursos humanos e as principais teorias da aprendizagem do século XX.

Palavras-chave: Aprendizagem Organizacional, Ambiente Virtual de Aprendizagem Personalizados, Inovação, Estilos de Aprendizagem

\begin{abstract}
The search for innovation in organizational spaces, with the use of personalized Virtual Learning Environments, implies explaining the processes responsible for behavioral modeling that has an impact on the experiences of employees. In this sense, the objective is to understand the influences of classical theories of learning of the 20th century on theories of management. This objective is complemented by exploring how to assist the organizational learning process with the use of virtual learning systems that adapt to users' learning styles and with a friendly interface. The method adopted was the narrative review of the literature collected from the bibliographic survey on the fields of learning theories and business schools. The main result is a dialogical analysis of the epistemological discourses that fuse the assumptions of human resource management schools and the main learning theories of the 20th century.
\end{abstract}

Keywords: Organizational Learning, Personalized Virtual Learning Environment, Innovation, Learning Styles

\title{
1 INTRODUÇÃO
}

A retomada de uma economia global está intrinsicamente relacionada ao aumento do fluxo de informação e à emergência de um leque maior de oportunidades de negócio. Logo, o compartilhamento de dados é o agente desencadeador da inevitabilidade de empresas disporem de mecanismos que garantam seu o alcance e recebimento, conforme Cossul e Frozza (2017), aspectos essenciais para que permaneçam competitivas no modelo de mercado capitalista.

Nesta perspectiva, o problema desta pesquisa está voltado para o desenvolvimento de um Sistema Virtual de Aprendizagem Personalizado capacitado para otimizar processos de Aprendizagem Organizacional e Clima de Inovação. Estes sistemas têm como função transmitir informações e auxiliar na construção do conhecimento e tomada de decisões (SUÁREZ, ARENCIBIA e PÉREZ, 2016). A sua estruturação ocorre mediante utilização de aspectos da Inteligência Artificial, que de acordo com Bayrakdar \& Çalhan (2018) é uma área da Computação que permite a execução de atividades pautadas em instruções semelhantes ao funcionamento do cérebro humano.

Tendo como base os fundamentos conceituais apresentados, bem como o problema de pesquisa que surge diante de transformações no modo como a sociedade do século XXI se organiza, apresenta-se como objetivo compreender as relações existentes entre as Teorias Clássicas de Aprendizagem do século XX e Teorias da Administração de Recursos Humanos. Para tornar o Sistema Virtual de Aprendizagem 
Personalizado eficaz é necessário compreender as origens e os efeitos da epistemologia presente nessa relação uma vez que são refletidos e reproduzidos no comportamento de colaboradores (as).

Epistemologicamente, o conhecimento é produzido, compartilhado e situado em determinado contexto espaço-temporal (GAYTÁN, 2020). A escolarização é compreendida como mecanismo responsável pelo ajustamento comportamental, garantindo reprodução de concepções arraigadas na nossa cultura (GUZZO, MOREIRA e MEZZALIRA, 2011). Paralelamente, os modos de produção, tais como o Fordismo, Taylorismo e Toyotismo, no século XX, colocam em prática técnicas de gerenciamento e aproveitamento do tempo e da tecnologia, com a intenção de aumentar a produtividade (WITCZAK, 2007).

\section{DESCRIÇÃO DO PROJETO}

A fim de contextualização, este estudo surge como atividade integrante do projeto " $\mathrm{O}$ uso de estilos cognitivos e de agentes pedagógicos no processo de ensino-aprendizagem em sistemas virtuais de aprendizagem" que representa um esforço conjunto dos Departamentos de Ciências da Saúde; Engenharias, Arquitetura e Computação; Ciências, Humanidades e Educação; e do Programa de PósGraduação em Sistemas e Processos Industriais. Este projeto abarca três linhas de pesquisa: a primeira é a linha de Aplicações de Técnicas de Inteligência Artificial na Gestão do Conhecimento e no processo de ensino-aprendizagem em Organizações; a segunda é denominada de Estudos Experimentais dos Processos Cognitivos e Comportamentais, visto que implica no funcionamento dos processos relativos ao raciocínio e à aplicação deste conhecimento no campo da educação e da informática; a terceira linha se refere ao Monitoramento, à Simulação e à Otimização de Sistemas e Processos.

Com base na interdisciplinaridade apresentada, foi desenvolvido o protótipo de um sistema virtual de aprendizagem personalizado, apoiado pelo uso do agente pedagógico Dóris, conforme ilustra a Figura 1, capacitado para extrair características individuais dos usuários, a medida em que estes interagem com a plataforma de ensino-aprendizagem (COSSUL et al., 2018). Este agente é conversacional e expressa emoções no processo de interação. Dóris possui três módulos: i) Perceptivo, que coleta informações da interação do usuário com o ambiente virtual; ii) Reativo, que ativa as expressões faciais de Dóris; iii) Cognitivo, que toma das decisões referente ao processo de interação. Além disso, a personalização deste modelo computacional, ocorre com base no estilo de aprendizagem do usuário de acordo com Felder \& Silverman (1988). Nesse caso, táticas de ensino, adequadas ao estilo identificado, são redirecionadas ao usuário, atribuindo qualidade à interação. Computação afetiva e critérios de usabilidade são utilizados, promovendo um ambiente motivacional, agradável e de fácil utilização. 
FIGURA 1: AGENTE DÓRIS

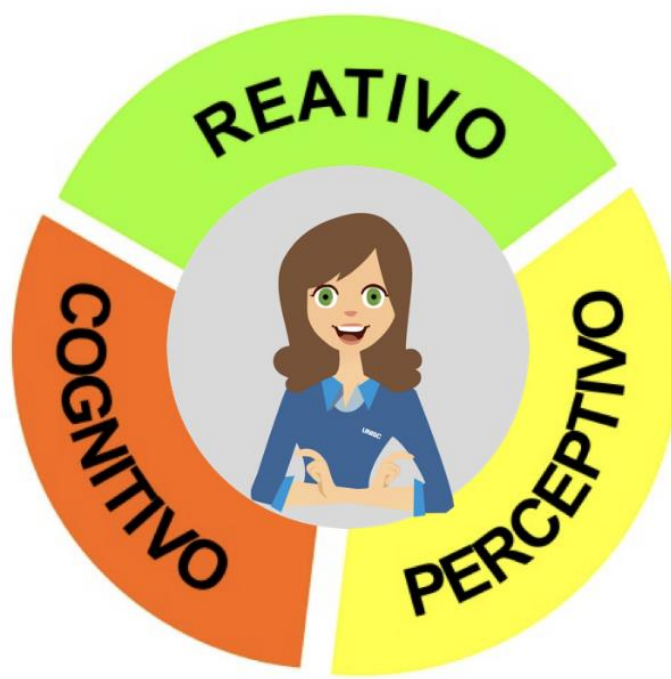

Fonte: (COSSUL et al., 2018).

A proposta é aplicar este modelo computacional em Organizações diversas, além das Instituições de Ensino. As atribuições deste modelo tendem a auxiliar em processos de aprendizagem organizacional e na mediação do clima para inovação.

\section{FUNDAMENTAÇÃO TEÓRICA}

A realização deste estudo, bem como o desenvolvimento do projeto com um todo, ocorrem mediante utilização dos conceitos "usabilidade", "aprendizagem organizacional" e "clima para inovação". Nesta seção, são expressas suas atribuições teóricas.

\subsection{USABILIDADE}

A usabilidade objetiva elaborar interfaces capazes de permitir uma interação eminentemente facilitada e eficaz entre sistema e usuário. Segundo Nielsen (1993), há cinco critérios básicos que devem ser alcançados em uma interface, a saber:

1- Intuitividade: O sistema deve apresentar facilidade de uso, permitindo que um usuário sem experiência seja capaz de produzir satisfatoriamente suas atividades.

2- Eficiência: O sistema deve ser eficiente em seu desempenho, apresentando um alto nível de produtividade.

3- Memorização: Suas telas devem apresentar facilidade de memorização, permitindo que usuários ocasionais consigam utilizá-las, mesmo depois de um longo intervalo de tempo navegando pelo ambiente. 4- A quantidade de erros apresentada pelo sistema deve ser o mais reduzido possível. Além disso, devem ser apresentadas soluções simples e rápidas. Erros graves ou sem solução não podem ocorrer. 
5- Satisfação: O sistema deve proporcionar interação agradável ao usuário.

Para analisar a usabilidade do ambiente, se faz necessário um acompanhamento da interação do usuário. Essas análises permitem constatar problemas, possibilidade de calcular métricas, diagnosticar características que possam atrapalhar a interação por desconformidade com padrões explícitos e implícitos, prever dificuldades de aprendizado na operação do sistema, prever os tempos de execução de tarefas, conhecer a opinião do usuário em relação ao sistema e sugerir as ações do reprojeto mais evidentes diante dos problemas de interação efetivos ou diagnosticados (CYBIS, 2007).

\subsection{APRENDIZAGEM ORGANIZACIONAL}

Processos de Aprendizagem Organizacional na contemporaneidade, relacionam-se com as capacidades criativas e produtivas dos colaboradores(as). Para que ela seja efetivada, cinco características devem ser contempladas pelo cenário empresarial, a saber: resolução sistêmica, experimentação, experiências passadas, circulação do conhecimento e benchmarking, e disciplina, que se referem ao domínio pessoal (autoconhecimento), a modelos mentais (ideias retificadas), a visões partilhadas, à aprendizagem em grupo e ao pensamento sistêmico (SENGE, 2011).

Nonaka e Takeuchi (2002) explicam o processo de criação e uso do conhecimento nas organizações a partir do modelo denominado de Espiral do Conhecimento, estruturado pela conversão do conhecimento tácito em conhecimento explícito. Nesse caso, especificamente, a externalização de ideias é o mecanismo utilizado para que ocorra a conversão, enquanto que a socialização serve para impulsionar o processo criativo das ideias (OLIVEIRA et al., 2010).

Sequeira (2008) descreve que o conhecimento tácito envolve duas dimensões - técnica e cognitiva (modelos mentais, crenças e percepções, de difícil transmissão) - e o conhecimento explícito, produzido a partir de dados recolhidos e armazenados. A combinação destas duas categorias de conhecimento emerge quatro modos de conversão do conhecimento, a saber: socialização, externalização, combinação e internalização. Por meio destes modos de conversão, os conhecimentos tácito e explícito se difundem em quantidade e qualidade gerando um ciclo ascendente do indivíduo até à interação com a organização e com o ambiente (NONAKA \& TAKEUCHI, 2002).

A obra de Pratkanis (1989) elucida o modelo sociocognitivista. Este modelo demonstra como indivíduos desenvolvem o seu potencial e contribuem para a socialização do conhecimento produzido. Nesse processo, utilizam-se das três partes constituintes da estrutura das atitudes: i) avaliações pessoais de um objeto de pensamento: categorização do objeto; ii) síntese avaliativa; e, iii) estrutura do conhecimento. A junção destes componentes tem por função gerar produção de sentido, isto é, a conversão do mundo da experiência em um mundo inteligível. 
O processo de sensemaking é considerado como a atividade central, tanto na construção da organização como dos ambientes com os quais se interage. Se sensemaking significa atribuir um novo significado para algo que já ocorre nos processos de organizar, mas que ainda não fora nomeado, ele consiste no mecanismo que gera os componentes do mundo organizacional (WEICK, 1995). As organizações por si só, enquanto engrenagens sólidas não são capazes para gerar conhecimento. Elas dependem da experiência subjetiva e intersubjetiva dos colaboradores. De acordo com Scatolin (2015), o sujeito que interage dentro do grupo, que apresenta iniciativa, produz o conhecimento fundamental para a empresa se manter competitiva.

\subsection{CLIMA PARA INOVAÇÃO}

Ferramentas e escalas utilizadas para diagnosticar o clima organizacional são consideradas mecanismos estratégicos essenciais para o gerenciamento de recursos humanos. Estes mecanismos possibilitam diagnosticar e criar condições voltadas para a gestão da qualidade de vida no trabalho mediante a identificação da origem dos problemas relacionados aos colaboradores.

Definir os aspectos referentes ao clima organizacional de determinada organização possibilita criar um plano de ação que vise a melhoria contínua (BENETTI et al., 2012). O século XXI está marcado por um cenário competitivo emergente, no qual informações são produzidas em escala e compartilhadas instantaneamente. Logo, para que as empresas se mantenham em vantagem competitiva, o capital intelectual deve ser gerenciado de modo a impulsionar a inovação e melhorias nos processos de tomada de decisão e desempenho (FÁTIMA, JUNIOR e JUNIOR, 2015).

O mecanismo mais utilizado para realizar o diagnóstico do clima organizacional é o SOQ (Situational Outlook Questionnairel), proposto por Ekvall em 1996. Composto por 10 fatores, a saber: alegria e dinamismo, conflitos, confiança e abertura, correr riscos, desafio e motivação, debates, ludismo e humor, liberdade, suporte às ideias e tempo para ideias. Cada fator é composto por 5 questões, que são aplicas de acordo com a demanda do local analisado (EKVALL, 1996).

O método contemplado, neste projeto, para realizar a análise dos dados referentes ao clima organizacional, é o método de análise multivariada HCA (Hierarchical Clustering Analysis - Análise por Agrupamento Hierárquico). As aplicações do HCA são diversas e abrangem vários ramos do conhecimento como a Psicologia, Ciências Sociais e Biológicas, Geologia, Química, Engenharia e outros (MINGOTI, 2005, ROSÁRIO; KIPPER; FERRÃO, 2012). O HCA interliga as amostras por suas associações, produzindo um dendrograma, na qual amostras semelhantes são agrupadas. O parâmetro considerado neste processo é a medida de distância entre as amostras, ou seja, quanto menor a distância entre as amostras, maior será a semelhança entre as mesmas; e quanto maior a distância, maior a diferença entre as amostras (MINGOTI, 2005). 


\section{METODOLOGIA}

Neste estudo, é possível explorar reflexões acerca da epistemologia presente na relação "Escolas de Administração e Recursos Humanos'versus “Teorias da Aprendizagem”, ambos conceitos do século XX. São considerados, para além desses conceitos, àqueles que relacionam-se com o problema da pesquisa e à fundamentação do projeto. Para o desenvolvimento, foi utilizado o método de pesquisa bibliográfica que consiste no levantamento da produção sobre determinado tema ou assunto (RUIZ, 1992).

Como base, foram privilegiadas referências de autores consagrados da literatura, tais como: Nonaka e Takeuchi (2002), Pratkanis (1989), Ekvall (1996). A originalidade de suas teorias repercute sócio e historicamente, contribuindo para a compreensão de diferentes fenômenos. Nesse sentido, compreende-se a ciência como um mecanismo que busca compreender experiências cotidianas que, embora sejam de tempos distantes, possuem efeitos que coexistem nos dias de hoje (NUNES, 2015).

Além disso, utilizar pioneiros em determinadas linhas de pesquisa, implica considerar evoluções e transformações sobre pontos de vista e teorizações (NUNES, 2015). Nesse sentido, a fim de somar estudos recentes, foi realizada uma busca no portal de periódicos da CAPES com os termos: "teorias da aprendizagem", "aprendizagem organizacional", "taylorismo", “fordismo", "toyotismo", “ambiente virtual de aprendizagem" e "usabilidade".

\section{RESULTADOS}

A realização de um estudo comparativo entre as escolas de administração de recursos humanos e as principais teorias da aprendizagem é o principal resultado alcançado. Seguem as correlações:

$1^{\circ}$ ) O Taylorismo, que apresenta correlações diretas com as teorias do condicionamento clássicas de Pavlov (1927) e os modelos experimentais de análise do comportamento de Watson (1919) e Skinner (1957). Isto porque, este modelo de produção privilegiava a tarefa repetitiva, a separação entre concepção e execução do trabalho e a alienação como resultado final deste processo.

$2^{\circ}$ ) O Fordismo pode ser alinhado à perspectiva da aprendizagem social ou vicária de Albert Bandura (que pressupõe o aprendizado pela observação das suas consequências) e de Vygotsky (1978), que buscou as origens sociais das capacidades humanas. Devido à utilização do consumo de massa e da individualização dos salários como fonte de motivação, da utilização dos processos de lideranças situacionais e o incremento de novos padrões de comunicação organizacional.

$3^{\text {o }}$ O Toyotismo, como representante dos modelos pós-fordistas/ contingencialistas, alinha-se ao conceito de motivação (ambiente, forças internas e objeto fim) e os pontos de ancoragem de David Ausubel, a relação entre os fatos e as ideias de Bruner (1983), as múltiplas inteligências de Gardner (2010) e a teoria das expressões de Ekman (2011). Esta escola de produção tem como principal 
característica o gerenciamento por estresse, o engajamento das equipes e a busca constante por soluções aos problemas cotidianos de trabalho, privilegiando as alternativas individuais e a responsabilização coletiva por resultados

\section{CONCLUSÃO}

Este estudo buscou elucidou a relação estreita e cronológica entre as Teorias da Aprendizagem e as Escolas de Administração de Recursos Humanos do século XX, delineando a forma como se estruturou a evolução, tanto do trabalho, quanto das teorias da aprendizagem no decorrer do século XX.

$\mathrm{O}$ século XX caracteriza-se como rico em esforços que buscam compreender e explicar o fenômeno psicológico no que tange as capacidades da mente humana (HARRÉ, 2016). A evolução referente aos modelos de produção estão intrinsicamente relacionados com o modo como os sujeitos aprendem e tornam-se produtivos nas organizações. Logo, podemos inferir que a escolarização é o mecanismo que ancora e arquiteta os modos de produção ao longo dos anos.

Compreender a existência da relação estreita e cronológica entre esses dois grandes campos do conhecimento, bem como os diversos modos de se pensar e executar as rotinas de trabalho, assim como os diferentes estilos de aprendizagem, elucida possibilidades de melhorias no modelo computacional desenvolvido.

Preparar Ambientes Virtuais de Aprendizagem Personalizados, em função das características de estilos de aprendizagem dos(as) colaboradores(as) em Organizações, a partir de uma interface com aspectos de usabilidade e uso de agente conversacional na interação, pode promover a melhoria contínua dos processos e atividades realizadas pelas pessoas com novas capacitações que respeitem o ritmo e o tempo disponível, além de incentivar a produção e o compartilhamento do conhecimento organizacional gerado. 


\section{REFERÊNCIAS}

BASTOS, A. V. B. Cognição nas organizações de trabalho. In: ZANELLLI, J. C.; BORGES-ANDRADE, J. E. BASTOS, A. V. B. (orgs). Psicologia, organizações e trabalho no Brasil. Porto Alegre: Artmed. 2004.

BENETTI, J. C.; KIPPER, L. M.; SILVA, R. B. DA; MARDER, L.; GRUNEVALD I. Use of Innovation Radar and Hierarchical Cluster Analysis for the Diagnosis of Organization Climate and Innovation. Anais do XVIII ICIEOM 2012 - Guimarães - Portugal. 2012.

BAYRAKDAR, M. E. ÇALHAN, A. Avaliação comparativa de desempenho de métodos eficientes de transferência de espectro em redes cognitivas sem fio. ANAIS da $26^{a}$ Conferência de Aplicativos de Processamento e Comunicação de Sinal 2018. doi: https://doi.org/10.1109/SIU.2018.8404573,

BRUNER, J. S. Child's Talk. New York: W.W. Norton, 1983.

COSSUL, D.; FROZZA, R.; FAGUNDES, B. J.; FERREIRA, G.; KIPPER, L. M.; WITCZAK, M. V. C. Evolução do agente pedagógico emocional Dóris em um ambiente virtual de aprendizagem. In: Editora Poisson. (Org.). Gestão da Produção em Foco. 1ªed. Belo Horizonte: Poisson, Vol. 24, p. 28-38. 2018.

COSSUL, D.; FROZZA, R. Inteligência Artificial Aplicada em Processos de Gestão Organizacional. In: ANAIS do XIII Congresso Nacional de Excelência em Gestão. Rio de Janeiro: Latec, 2017. v. 8. p. 1-10.

CYBIS, W.; BETIOL, A. H.; FAUST, R. Ergonomia e usabilidade: Conhecimentos, métodos e aplicações. São Paulo: Novatec, 2007. 344 p.

EKMAN, P. A linguagem das emoções. 3. ed. São Paulo: Lua de Papel, 2011.

EKVALL, G. Organizational climate for creativity and innovation. European Journal of Work and Organizational Psychology, v. 5: 1, 1996, pp. 105-123, DOI: 10.1080/13594329608414845

FÁTIMA, A. C. de; NASTASI JUNIOR, E.; LIMA JUNIOR, F. R. Uma ferramenta para avaliação do nível de maturidade da gestão do conhecimento organizacional. Revista Eletrônica Gestão \& Saúde. Vol. 6 (Supl. 2). Abril, 2015 p.873-90.

FELDER, R. M.; SILVERMAN, L. K. Learning and teaching styles in engineering education. Engineering education, 1988, pp. 674-681.

GARDNER, H.; CHEN, J.; MORAN, S. Inteligências Múltiplas ao redor do mundo. São Paulo: Artmed, 2010.

GAYTÁN, J. F. Á. Epistemología de la ciencia y apertura para la investigación educativa. Voces de la educación, 01 January 2020, Vol.5(9), pp.142-148.

GUZZO, R. S. L.; MOREIRA, A. P. G.; MEZZALIRA, A. S. C. Avaliação Psicossocial: Desafios para a prática profissional nos contextos educativos. Avaliação Psicológica 10(2), 163-171. 2011.

MINGOTI, S. A. Análise de dados através de métodos de estatística multivariada: uma abordagem aplicada. Belo Horizonte: Ed. da UFMG, 2005.

NIELSEN, Jakob. Usabilty Engineering, Morgan Kaufmann: San Francisco, 1993.

NONAKA, I.; TAKEUCHI, H. Criação de conhecimento na empresa: como as empresas japonesas geram a dinâmica da inovação. 10. ed. Rio de Janeiro: Campus, 2002. 
NUNES, E. D. Dos clássicos na literatura aos clássicos na sociologia e na sociologia médica/saúde. Physis Revista de Saúde Coletiva, Rio de Janeiro, 25 [2]: 401-421, 2015 Doi: http://dx.doi.org/10.1590/S0103-73312015000200005

OLIVEIRA, M.; BECKER, G. V.; PEDRON, C. D.; IGNA, F. D. Espiral do conhecimento em frameworks de gestão do conhecimento: o caso de duas organizações em Portugal. Perspect. ciênc. inf. vol.15 no.3 Belo Horizonte, 2010. Doi: https://doi.org/10.1590/S1413-99362010000300009

PAVlov, I. Conditioned Reflexes: The Investigation of the Physiological Activity of the Cerebral Cortex. London: Oxford University Press, 1927.

PRATKANIS, A. R. The cognitive representation of attitudes. In A. R. Pratkanis, S. J. Breckler, \& A. G. Greenwald (Eds.). The third Ohio State University Vol. on attitudes and persuasion. Attitude structure and function (p. 71-98). Lawrence Erlbaum Associates, Inc. 1989.

ROSÁRIO, C. R.; KIPPER, L. M.; FERRÃO, M. F. Modelo para aplicação de análise multivariada através de técnicas estatísticas PCA e HCA sobre questionário de avaliação da satisfação de clientes: Estudo de caso em uma empresa metalúrgica de embalagens metálicas. Tecno-Lógica, v. 16, n. 1, p. 3039, 2012.

RUIZ, J.A. Metodologia científica: guia para eficiência nos estudos. São Paulo (SP): Atlas; 1992.

SCATOLIN, H. G. A gestão do conhecimento nas organizações: o legado de Nonaka e Takeuchi. Perspectivas Em Gestão \& Conhecimento, 5(2), 4-13, 2015. Recuperado de https://periodicos.ufpb.br/ojs2/index.php/pgc/article/view/21772

SENGE, P. M.; ZIDE NETO, A quinta disciplina: arte e prática da organização de aprendizagem. 27. ed. São Paulo: Best Seller: 2011.

SEQUEIRA, B. Aprendizagem Organizacional e a Gestão do Conhecimento: uma abordagem multidisciplinar. ANAIS do VI Congresso Português de Sociologia, Mundos Sociais: saberes e práticas. Lisboa, 25 a 28 de junho de 2008. http://www.aps.pt/vicongresso/pdfs/497.pdf

SKINNER, B. F. Verbal Behavior. London: Methuen, 1957.

VYGOTSKY, L. S. Mind in Society. Cambrige, MA: Harvard University Press, 1978.

WATSON, J. B. Psychology from the Standpoint of a Behaviorist. Philadelphia: Lippincott, 1919.

WITCZAK, M. V. C. Dos cenários e dos atores - trabalho, destruição e consumo em dois tempos: da Modernidade a Pós-Modernidade. IN: VERONESE, M. V.; GUARESCHI, P. A. G. Psicologia do Cotidiano - Representações Sociais em ação. Rio de Janeiro: Vozes Editora, 2007. 311 p.

WEICK, K. E. Sensemaking in organizations. Thousand Oaks: Sage Publications. 1995. 231 p. 